Revista Energia na Agricultura

ISSN 1808-8759

\title{
ANÁLISE MATEMÁTICA E ESTATÍSTICA DA PRODUTIVIDADE DE LAVOURAS CAFEEI- RAS AGROQUÍMICA E ORGÂNICA NA REGIÃO DA ALTA PAULISTA ${ }^{\mathbf{1}}$ \\ JOSÉ EDUARDO FERREIRA GABRIEL ${ }^{2}$; LUÍS ROBERTO ALMEIDA GABRIEL FILHO ${ }^{3}$; CAMILA PIRES CREMASCO ${ }^{4} \&$ ELIAS JOSÉ SIMON ${ }^{5}$
}

RESUMO: Estudos realizados com sistemas orgânicos têm mostrado a viabilidade e sustentabilidade ecológica e social desses agroecossistemas, ao contrário dos sistemas agroquímicos (convencionais) de produção. Alguns estudos concluíram que o modelo agroquímico existe pouca interação entre os fluxos de energia interna, basicamente a lavoura recebe todos os insumos para a produção não havendo aumento na "qualidade energética" interna ao sistema, enquanto que no modelo orgânico de produção apresenta maior interação entre os diferentes recursos existentes no sistema. As atuais crises econômica e ecológica, expõem a insustentabilidade do padrão produtivo da agricultura desenvolvida de forma industrializada, evidenciando à dependência dos países do primeiro mundo na importação de commodities agrícolas produzidas no terceiro mundo, dentre elas, o café. Diante destes fatos desenvolveu-se uma pesquisa para identificar os problemas na região da Alta Paulista, Oeste do Estado de São Paulo, com relação aos sistemas de produção de café. Atualmente, o problema fundamental, de acordo com a pesquisa realizada, dos agricultores nesta região, reside na escolha de um sistema de produção corretamente viável (ambiental, social e econômico); agroquímico ou orgânico. Os objetivos deste estudo foram analisar a produtividade dos sistemas de produção de café orgânico e agroquímico e no período de 2003 a 2007, em 30 propriedades produtoras, localizadas nesta região, com a finalidade de apontar o sistema de produção que apresente a maior produtividade. De acordo com a metodologia da CONAB, os dados coletados foram registrados em planilhas eletrônicas para serem utilizados como variáveis nos modelos de análises estatística e matemática. Realizou-se uma análise descritiva dos dados de produtividade e utilizou-se para análise estatística o testes paramétricos e não paramétricos para análise de variância. A análise matemática das curvas foram elaboradas com o software Origin for Windows 6.0, que utiliza métodos numéricos para ajuste dos dados fornecidos à uma função de parâmetros variáveis. Ao contrário dos sistemas convencionais de produção, o sis-

\footnotetext{
${ }^{1}$ Parte da tese de doutorado do $1^{\mathrm{o}}$ autor intitulada: Análise da produtividade e rentabilidade de lavouras cafeeiras agroquímica e orgânica na região da Alta Paulista

${ }^{2}$ Aluno do curso de PG Energia na Agricultura - FCA/UNESP - Botucatu/SP - Brasil. Docente e coordenador dos cursos de Administração e Ciências Econômicas - FAI - Adamantina/SP - Brasil. jefgabriel@yahoo.com.br

${ }^{3}$ Docente do curso de PG Irrigação e Drenagem - FCA/UNESP - Botucatu/SP e do curso de Administração CE/UNESP - Tupã/SP - Brasil. gabrielfilho@tupa.unesp.br

${ }^{4}$ Docente do curso Tecnologia em Agronegócio - FATEC - Presidente Prudente/SP. camila@fatec.edu.br

${ }^{5}$ Orientador e docente do Departamento de Gestão e Tecnologia Agroindustrial - FCA/UNESP - Botucatu/SP. ejsimon@fca.unesp.br
} 
tema orgânico evidenciou maior viabilidade do modelo de produção. Além disto, com a modelagem quantitativa proposta, é possível a realização da avaliação destes tipos de investimentos, proporcionando maior segurança ao agricultor, no momento da decisão.

Palavras-chave: Agroecossistemas, modelagem quantitativa, análises estatísticas e matemática.

\section{MATHEMATICS AND STATISTICAL ANALYSIS OF PRODUCTIVITY OF AGROCHEMI- CALS AND ORGANIC COFFEE PLANTATIONS IN ALTA PAULISTA REGION}

SUMMARY: Studies with organic systems have shown the feasibility and ecological and social sustainability of these agroecosystems, unlike the systems agrochemicals (conventional) production. Some studies have concluded that for the model agrochemical exists less interaction between the flow of internal energy, basically the crop receives all inputs to production with no increase in "energy quality" within the system, while in the organic model of production has increased interaction between different resources in the system. The current economic and ecological crisis, exposed no sustainability of the production pattern of industrialized agriculture developed in a way, showing the dependence of developed countries on imports of agricultural commodities produced in the third world, among there coffee. Given these facts, developed a survey to identify problems in the Alta Paulista region, west of São Paulo State, in relation to coffee production systems. Actually, the fundamental problem, according to the research, farmers in this region, is to choose a viable production system correctly (environmental, social and economic); agrochemical or organic. The objectives of this study were to analyze the yield of production systems and agro-chemical and organic coffee in the period from 2003 to 2007, in 30 producing properties, located in this region, in order to point the production system to produce the highest yield. According to the methodology of CONAB, data collected were recorded on spreadsheets to be used as variables in statistical analysis models and mathematics. We performed a descriptive analysis of productivity data and were used for statistical analysis tests for parametric and nonparametric analysis of variance. The mathematical analyses of the curves were prepared with Origin for Windows 6.0 software, which uses numerical methods to fit the data supplied to a function of variable parameters. Unlike conventional systems of production, the organic system showed greater viability of the production model. Furthermore, with the quantitative modeling proposal, it is possible to perform the evaluation of these types of investments, providing more security to the farmer at the time of decision.

Keywords: Agroecosystems, quantitative modeling, statistical analysis and mathematical. 


\section{INTRODUÇÃO}

A colonização do Estado de São Paulo teve como marco histórico a lavoura cafeeira, que foi responsável pela criação e desenvolvimento econômico e social de diversas cidades, por longo período. Atualmente, o espaço que foi ocupado por essa lavoura encontra-se em pleno retrocesso, principalmente pela opção da cultura da cana-de-açúcar.

As atuais crises econômica e ecológica, expõem a insustentabilidade do padrão produtivo da agricultura desenvolvida de forma industrializada, evidenciando à dependência dos países do primeiro mundo na importação de commodities agrícolas produzidas no terceiro mundo, dentre elas, o café. Agravando-se o problema no Brasil onde também existe uma demanda reprimida no mercado interno.

Esse fato vem chamando a atenção para a convergência de três grandes dilemas descritos por Petersen e Almeida (2008), com os quais a humanidade se depara: o primeiro se refere ao aumento exponencial dos preços do petróleo e suas implicações diretas sobre os custos dos agroquímicos. O segundo está ligado aos impactos ainda imprevisíveis das mudanças climáticas sobre a produção alimentar. O terceiro é a degradação e a perda em ritmos acelerados da agrobiodiversidade, dos solos e dos recursos hídricos em função do emprego de métodos predatórios de produção agrícola que vêm sendo subsidiados há décadas pela energia barata do petróleo.

Os desequilíbrios nos ecossistemas agrícolas provocados pela monocultura induziram os procedimentos técnicos que definem o chamado "pacote" tecnológico da agricultura moderna (BORGES FILHO, 2005). Segundo Romeiro (1998), na natureza, diversidade é sinônimo de estabilidade, e, quanto mais simplificado for um determinado ecossistema, maior a necessidade de fontes de energia para manter o equilíbrio.

Esse modelo de exploração agrícola de larga escala iniciado através da modernização da agricultura têm no bojo de seu processo produtivo um manejo insustentável e dificilmente poderão implementar as múltiplas dimensões da sustentabilidade (ecológica, econômica, social, cultural, ética e política) se não iniciarem uma mudança gradual no manejo das lavouras, nas relações sociais e econômicas existentes nas unidades de produção.

Ao contrário dos sistemas convencionais de produção, estudos realizados com sistemas orgânicos ou agroflorestais têm mostrado a viabilidade e sustentabilidade ecológica, social e econômica desses agroecossistemas, dadas principalmente, pela sua alta capacidade de resiliência, confiabilidade, autosuficiência e produtividade (MACEDO \& PEREIRA, 2000; PENEIREIRO et al. 2000; REIS \& HILDEBRAND, 2000).

Segundo Veiga (2003), pode-se dizer que todas as definições de agricultura sustentável transmitem a visão de um futuro padrão produtivo de alimentos, fibras e matérias-primas energéticas que garan- 
tam a manutenção, no longo prazo, dos recursos naturais e da produtividade agropecuária; o mínimo de impactos adversos ao ambiente; retorno adequado aos produtores; otimização da produção com um mínimo de insumos externos; satisfação das necessidades humanas de alimentos e renda; atendimento às demandas sociais das famílias e comunidades rurais.

Diante destes fatos, desenvolveu-se uma pesquisa para identificar os problemas na região da Alta Paulista, Oeste do Estado de São Paulo, com relação aos sistemas de produção de café. Atualmente, o problema fundamental, de acordo com a pesquisa realizada, dos agricultores nesta região, reside na escolha de um sistema de produção mais produtivo, agroquímico ou orgânico.

Dada a atual crise ecológica da cafeicultura convencional (agroquímica), o presente trabalho teve como objetivos analisar a produtividade, efetuar comparações dos sistemas de produção de café orgânico e agroquímico e realizar avaliações no período de 2003 a 2007, em 30 propriedades produtoras, localizadas nesta região (Alta Paulista, Oeste do Estado de São Paulo), com a finalidade de apontar o sistema de produção que apresente a maior viabilidade de produção ao produtor, para que as lideranças estaduais e municipais possam priorizar e ajustar os projetos às reais necessidades dos municípios paulistas, que produzem ou venham a produzir o café.

\section{MATERIAL E MÉTODOS}

Foram coletados dados em trinta propriedades produtoras de café, sendo quinze com sistema de produção agroquímico (convencional) e quinze com sistema de produção orgânico. Os dados registrados em planilhas eletrônicas, foram os resultados das colheitas, de diversas idades, dos anos de 2003 a 2007 (cinco anos). Vale ressaltar que, em cada propriedade, foi realizado o levantamento dos dados de cinco blocos de produção (áreas distintas), considerando as datas de plantios e colheitas, com idade determinada (4,5 a 8,5 anos). Na obtenção dos dados primários sobre a cafeicultura orgânica e tradicional (convencional - agroquímica), foram realizadas entrevistas, após o término da safra 2006/2007.

O estudo foi realizado em uma parte da região da Alta Paulista, localizada no oeste do estado de São Paulo que é formada pelas cidades de Garça, Gália, Vera Cruz, Marília, Alvinlândia, Lupércio, Álvaro de Carvalho, Marília, Tupã, Parapuã, Osvaldo Cruz, Lucélia, Adamantina e Junqueirópolis, entre outros municípios produtores (ASSOCIAÇÃO DOS PRODUTORES ORGÂNICOS DE GARÇA E REGIÃO, 2006).

As análises estatísticas descritiva dos dados (de produtividade) foram realizadas de acordo com análises de variância, teste F de Snedecor, para dados que apresentavam distribuição normal, com posterior comparação de médias pelo teste de Tukey. Para os dados com distribuição não-normal, utilizou-se o teste não paramétrico de Kruskal-Wallis, com posterior comparação de medianas pelo teste de Dunn (MATOS, 2000; GUJARATI, 2000). 
As análises matemáticas das curvas utilizadas neste trabalho foram elaboradas com o software $O$ rigin for Windows 6.0, que utiliza métodos numéricos para ajuste dos dados fornecidos a uma função de parâmetros variáveis. A função eleita para o ajuste dos modelos apresentados, caracteriza comportamento hiperbólico e possibilita translações nos eixos das ordenadas e abscissas.

\section{RESULTADOS E DISCUSSÃO}

\subsection{Análise Estatística}

A primeira análise do presente trabalho foi voltada à descrição da produtividade de dois sistemas cafeeiros: agroquímico e orgânico. Os dados, obtidos em 30 propriedades rurais (15 em cada sistema cafeeiro) com 5 amostras de blocos de produção por propriedade, compuseram um espaço amostral de $30 \times 5=150$ dados de produtividade por hectare. Como a idade dos cafezais variava entre 4,5 a 8,5 anos, foram criadas quatro classes de idade:

- Classe (I): cafezais com menos de 6 anos de idade, denotado por " $<6$ ";

- Classe (II): cafezais com 6 anos de idade (inclusive) a 7, denotado por "6 |- 7";

- Classe (III): cafezais com 7 anos de idade (inclusive) a 8, denotado por "7 $\mid-8$ ";

- Classe (IV): cafezais com mais que 8 anos de idade (inclusive), denotado por " $\geq 8$ ".

A análise descritiva dos dados foi realizada de acordo com o sistema cafeeiro e as classes de idades, conforme ilustram a Tabela 1 e a Figura 1 para a produtividade dos cafezais, que revelam um comportamento inverso. Crescimento da produtividade para sistema orgânico e decrescimento para sistema agroquímico.

Tabela 1 - Análise descritiva da produtividade de sacas de café por hectare nos blocos de produção das propriedades rurais dos sistemas cafeeiros agroquímico e orgânico no período de 2003 a 2007. Alta Paulista Oeste do Estado de São Paulo.

\begin{tabular}{cccccc}
\hline \multirow{3}{*}{ Sistemas Cafeeiros } & Classes & n & Média & Desvio Padrão & Mediana \\
\hline \multirow{3}{*}{ Agroquímico } & (I) & 10 & 19,75 & 0,448 & 19,65 \\
& (II) & 20 & 18,75 & 0,831 & 18,9 \\
& (III) & 35 & 18,11 & 0,621 & 18,1 \\
& (IV) & 10 & 18,00 & 0,309 & 18,1 \\
\hline \multirow{3}{*}{ Orgânico } & (I) & 25 & 16,40 & 0,908 & 16,2 \\
& (II) & 30 & 17,09 & 0,702 & 17,25 \\
& (III) & 15 & 18,00 & 0,846 & 18,14 \\
& (IV) & 5 & 18,50 & 1,063 & 18,6 \\
\hline
\end{tabular}




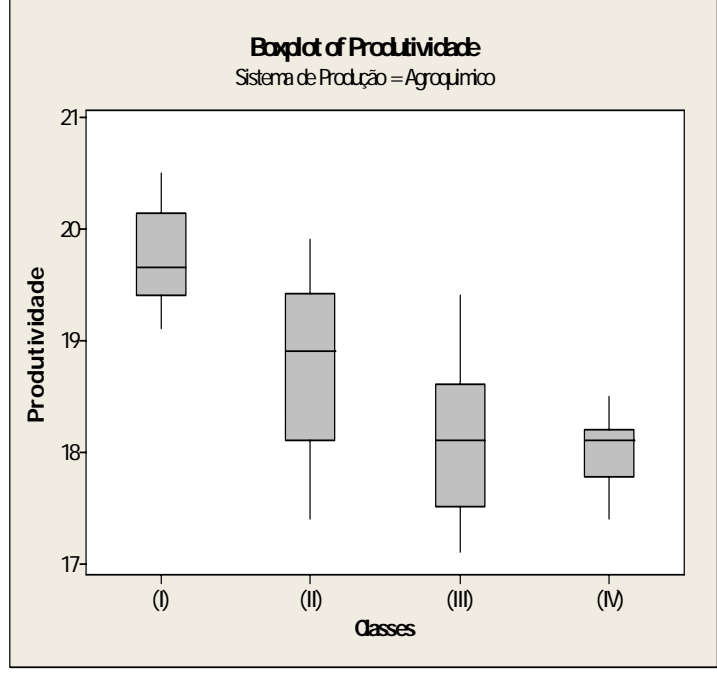

(i)

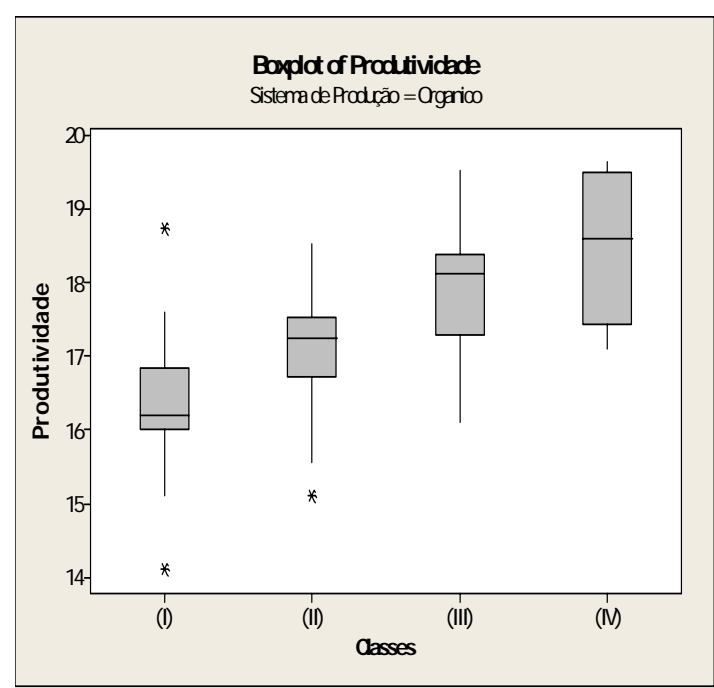

(ii)

Figura 1 - Boxplot para dados amostrais da análise da produtividade nos sistemas de produção Agroquímico (i) e Orgânico (ii) nas diferentes classes de idade dos cafezais (I, II, III, IV) relativamente aos blocos de produção das propriedades rurais em estudo.

A Tabela 2 apresenta as comparações realizadas com os p-valores seguidos da letra (F), quando obtido pelo teste F de Snedecor, e da letras (KW), quando obtido pelo teste de Kruskal-Wallis, totalizando 12 análises de variância efetuadas.

Tabela 2 - Valores médios e desvio padrão da produtividade de sacas de café por hectare dos blocos de produção das propriedades rurais em estudo obtidos nos sistemas cafeeiros (agroquímico e orgânico) nas classes de idade de cafezais consideradas (I, II, III, IV).

\begin{tabular}{cccccc}
\hline Sistemas Ca- & \multicolumn{5}{c}{ Classes } \\
\cline { 2 - 6 } feeiros & (I) & (II) & (III) & (IV) & $\boldsymbol{p}$ \\
\hline Agroquímico & $19,8 \pm 0,4 \mathrm{Aa}$ & $18,8 \pm 0,8 \mathrm{Aab}$ & $18,1 \pm 0,6 \mathrm{Ab}$ & $18,0 \pm 0,3 \mathrm{Ab}$ & $<0,001(\mathrm{KW})$ \\
Orgânico & $16,4 \pm 0,9 \mathrm{Ba}$ & $17,1 \pm 0,7 \mathrm{Bb}$ & $18,0 \pm 0,8 \mathrm{Ab}$ & $18,5 \pm 1,1 \mathrm{Ab}$ & $<0,001(\mathrm{KW})$ \\
$\boldsymbol{P}$ & $<0,001(\mathrm{~F})$ & $<0,001(\mathrm{~F})$ & $0,622(\mathrm{~F})$ & $0,356(\mathrm{~F})$ & - \\
\hline
\end{tabular}

Legenda: Caselas seguidas de letras distintas, maiúsculas nas colunas e minúsculas nas linhas, diferem entre si ao nível de significância de $5 \%$ pelo teste de Tukey, para as comparações com indicação (F), e pelo Teste de Dunn, para as comparações com indicação $(\mathrm{KW})$. Valores de $\mathrm{p}$ seguidos de $(\mathrm{F})$ indicam a utilização do teste paramétrico $\mathrm{F}$ de Snedecor (comparações de médias) e, seguidos de (KW), indicam utilização do teste não paramétrico de KruskalWallis, para comparação de medianas. 
A Tabela 2 confirma a hipótese de diferenças entre os sistemas cafeeiros em quase todas as classes, exceto nas classes (III) e (IV) para a produtividade. Além disto, a Tabela 2 revela, que o sistema agroquímico apresenta um decrescimento sensível na sua produtividade, com diferença significativa entre a produção da primeira (I) classe com as últimas (III e IV).

Frente aos resultados apresentados, é de grande interesse quantificar as possíveis diferenças entre os sistemas, seja na produção ao final dos 4 períodos (classes de idades), objetivando identificar o melhor sistema de produção em quantidade produzida. Para isto, foram construídas curvas que ajustem os valores obtidos para uma devida comparação dos resultados.

\subsection{Análise Matemática}

As curvas utilizadas neste trabalho foram elaboradas com o software Origin for Windows 6.0, que utiliza métodos numéricos para ajustes dos dados fornecidos à uma função de parâmetros variáveis. A função eleita para ajuste dos modelos apresentados, que caracteriza comportamento hiperbólico e possibilita translações nos eixos das ordenadas e abscissas.

Vale ressaltar que $f(x)$ será denotada por $P(x)$ quando a modelagem for realizada para a produção. Os valores de $x$, desta forma, existem somente nos pontos $x=5, x=6, x=7$ e $x=8$, e este presente ajuste de curvas objetiva, inicialmente, criar uma lei que descreva a produção de cada sistema cafeeiro em tais pontos.

Para o ajuste dos parâmetros $a, b$ e $c$, utilizaram-se como valores iniciais no sistema os pontos dados pelas médias apresentadas na Tabela 2. Importa o fato de que tais pontos representam apenas as condições iniciais do sistema, que realiza várias iterações até alcançar um ajuste adequado estatisticamente, via teste qui-quadrado.

Visto que pela Tabela 2 não há diferença estatística significativa entre os valores de produtividade nas classes (III) e (IV) ( $x=7$ e $x=8$ ), optou-se por utilizar para a inserção das condições iniciais os pontos associados à $x=5, x=6$ e $x=7$, uma vez também que somente três pontos seriam necessários e suficientes para a determinação dos parâmetros $a, b$ e $c$. O sistema proposto foi baseado nas seguintes equações:

$$
f(x)=a+\frac{b}{c+x}, x=5,6,7 \Rightarrow\left\{\begin{array} { l } 
{ f ( 5 ) = a + \frac { b } { c + 5 } } \\
{ f ( 6 ) = a + \frac { b } { c + 6 } } \\
{ f ( 7 ) = a + \frac { b } { c + 7 } }
\end{array} \Rightarrow \left\{\begin{array}{l}
a=f(5)-\frac{b}{c+5} \\
b=(c+6) \cdot(f(6)-a) \\
c=\frac{b}{f(7)-a}-7
\end{array}\right.\right.
$$


A solução do sistema (1) é determinada através do software Mathematica 5.2 atribuindo-se anteriormente os valores de $f(5), f(6)$ e $f(7)$, e utilizando os comandos seguintes:

$$
\begin{gathered}
F 5=19.8 ; F 6=18.8 \text { e } F 7=18.1 \\
\text { eqns }=\{a==F 5-b /(c+5), b==(c+6) *(F 6-a), c==(b /(F 7-a))-7\} ; \text { Solve }[\text { eqns },\{a, b, c\}]
\end{gathered}
$$

que ilustram um dos sistemas propostos (caso Produtividade / Agroquímico), retornando o seguinte resultado:

$$
\{\{b \rightarrow 26.4444, a \rightarrow 14.1333, c \rightarrow-0.333333\}\}
$$

O resultado das demais simulações, retornando valores iniciais para posterior simulação numérica de ajuste de curvas, juntamente com os valores médios de produtividade a serem associados nos pontos $x=5, x=6$ e $x=7$ estão dispostos na Tabela 3.

Tabela 3 - Valores médios da produtividade de sacas de café por hectare e estimativas de valores iniciais dos parâmetros $a, b$ e $c$.

\begin{tabular}{ccccccc}
\hline Sistemas Cafeeiros & $f(5)$ & $f(6)$ & $f(7)$ & $a$ & $b$ & $c$ \\
\hline Agroquímico & 19,8 & 18,8 & 18,1 & 14,13 & 26,44 & $-0,33$ \\
Orgânico & 16,4 & 17,1 & 18 & 10,80 & $-50,4$ & -14 \\
\hline
\end{tabular}

É importante ressaltar que foram somente necessários três pontos de médias para determinar os três parâmetros $a, b$ e $c$, não havendo necessidade de utilizar um $4 .^{\circ}$ ponto. Além disto, este ponto que seria dado por $f(8)$ é estatisticamente igual ao penúltimo, segundo as análises de variância realizadas, vindo a corroborar com sua não utilização. Por fim, a utilização somente de médias foi importante para a padronização dos cálculos e sua utilização nas funções e estimativas de parâmetros, descartando a utilização de medianas que foram utilizadas em algumas das comparações não paramétricas com o teste de Kruskal-Wallis.

Utilizando o software Origin, foi possível, inserindo as médias de cada período de tempo (Tabela 2) e os parâmetros iniciais $a, b$ e $c$ (Tabela 3), ajustar a função (I) para cada sistema cafeeiro e cada variável do presente estudo (produtividade). A Tabela 4 ilustra os resultados dos parâmetros da função (1) para cada um destes casos. 
Tabela 4 - Valores dos parâmetros $a, b$ e $c$ e funções de produtividade $P(x)$ associadas.

\begin{tabular}{cccc}
\hline Sistemas Cafeeiros & $a$ & $b$ & $c$ \\
\hline Agroquímico & 16,8 & 5,3 & $-3,2$ \\
Orgânico & 19,8 & $-7,7$ & $-2,8$ \\
\hline
\end{tabular}

Substituindo os valores da Tabela 4 na equação (1), é possível obter as seguintes equações que modelam matematicamente as médias obtidas na Tabela 2 para o sistema agroquímico:

$P(x)=16,82+\frac{5,27}{-3,23+x}$,

e o sistema orgânico:

$P(x)=19,82+\frac{-7,72}{-2,78+x}$

Os gráficos das funções (2) e (3) são dadas pelas Figuras 2 e 3, enquanto que a Figura 4 ilustra as intersecções destes gráficos de produtividade.

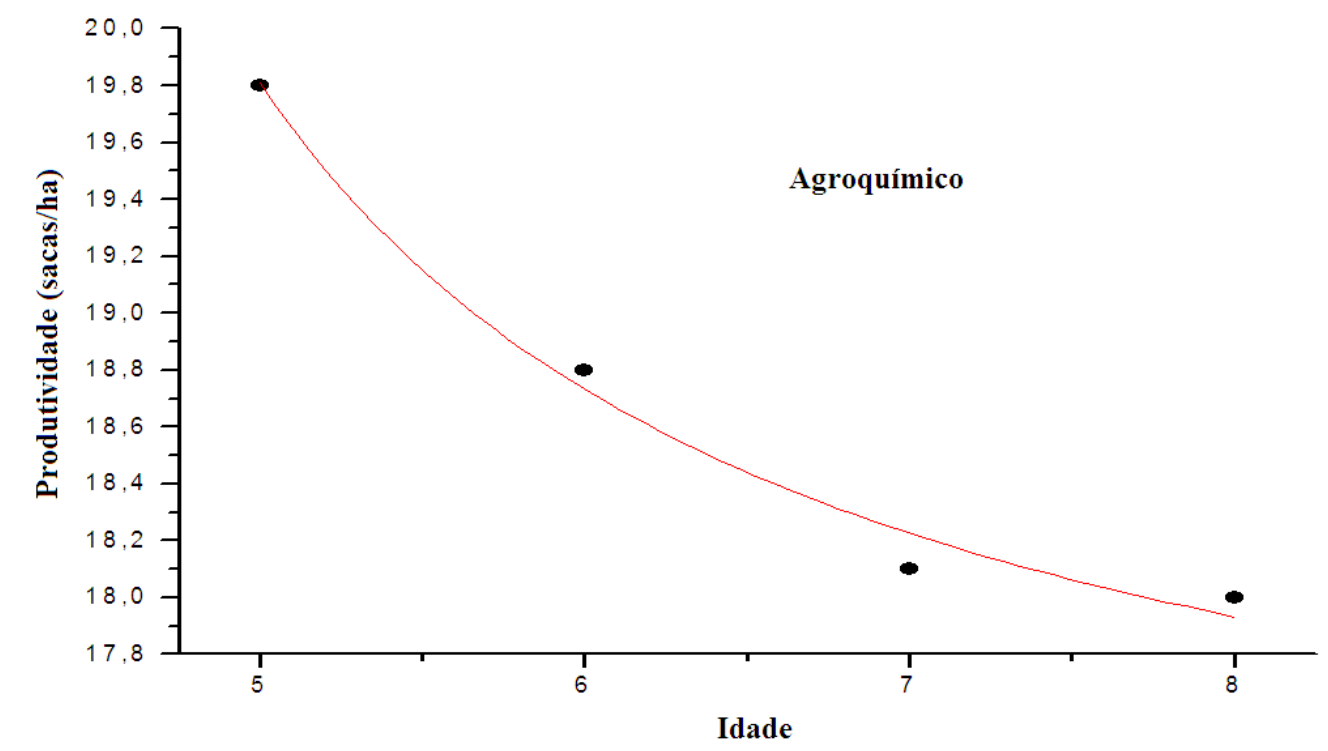

Figura 2 - Gráfico da função de produtividade do sistema cafeeiro agroquímico 


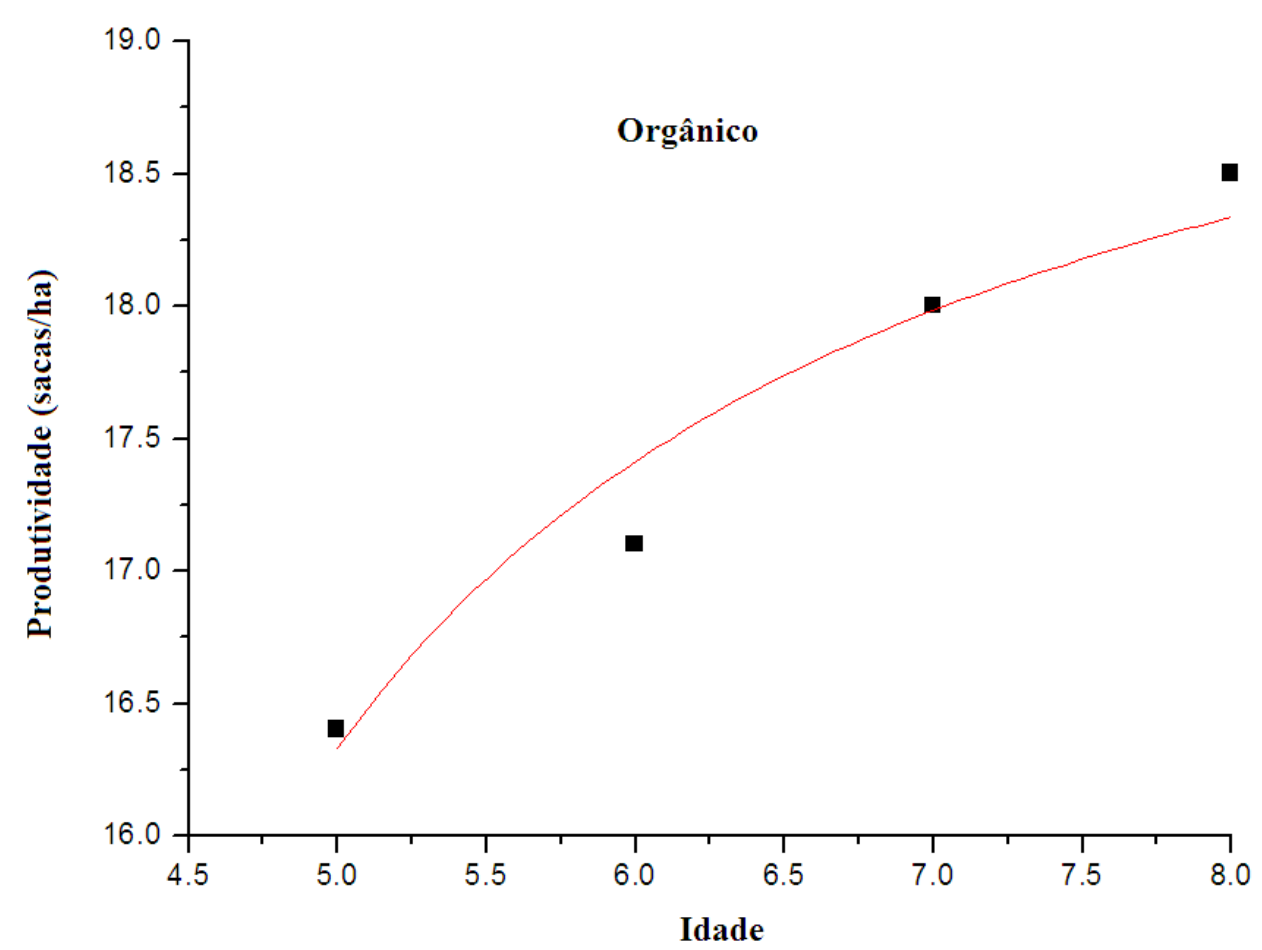

Figura 3 - Gráfico da função de produtividade do sistema cafeeiro orgânico.

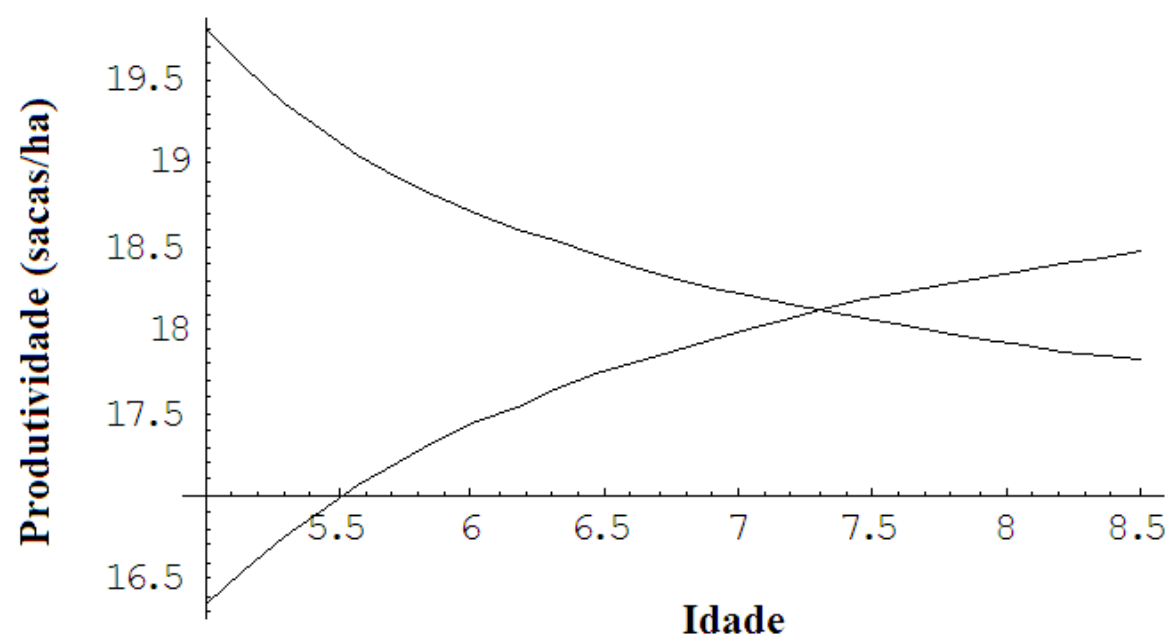

Figura 4 - Funções de produtividade realizadas pelo software Mathematica com o comando $\operatorname{Plot}[\{16.82+(5.27 /(-3.23+x)), 19.82+(-7.72 /(-2.78+x))\},\{x, 5,8.5\}]$.

Através da Figura 4, é possível verificar que o sistema agroquímico ao longo do tempo apresenta redução de produtividade, enquanto o orgânico apresenta aumento. Observa-se ainda, que a produtividade 
dos cafezais com idade acima de 7,5 anos, baseada no sistema orgânico tende a ultrapassar o sistema agroquímico.

\subsection{Produtividade acumulada dos sistemas cafeeiros}

A produtividade acumulada de um sistema cafeeiro até um determinado ano $n$ (idade do cafezal) é definida através da agregação da quantidade de sacas produzida anualmente por hectare desde o primeiro ano de estudo até o ano $n$.

Utilizando os valores médios constantes na Tabela 2, tem-se para o sistema agroquímico:

$P_{A}=\sum_{t=4}^{n} P_{t}=74,6$,

e o sistema orgânico:

$P_{A}=\sum_{t=4}^{n} P_{t}=70$

Desta forma, verifica-se que o sistema de produção agroquímico apresentou produtividade média, no período estudado, de 74,6 sacas por hectare, enquanto que o sistema de produção orgânico apresentou 70 (sacas/hectare). Portanto, pode-se afirmar que o sistema de produção agroquímico apresentou uma produtividade acumulada maior que o orgânico.

\section{CONCLUSÕES}

De acordo com as análises estatísticas e os modelos matemáticos, o sistema agroquímico de produção de café analisado em 15 pequenas propriedades rurais demonstrou que a idade dos cafezais influenciou sua produtividade. A idade dos cafezais é responsável pela variação da produtividade e a produtividade dos cafezais agroquímicos diminuiu de acordo com a sua idade.

Para o sistema orgânico de produção, que também teve 15 pequenas propriedades rurais analisadas, demonstrou-se que a idade dos cafezais influenciou a produtividade. A idade também é responsável pela variação da produtividade, e a produtividade dos cafezais orgânicos aumentou de acordo com a sua idade.

Portanto, as produtividades dos cafezais agroquímicos diminuem de acordo com a sua idade, enquanto que as dos cafezais orgânicos aumentam.Tal fenômeno deve-se, possivelmente, ao fato do sistema de produção orgânico ser desenvolvido com base em recursos naturais. Confirmando o estudo realizado 
por Ortega (2002), que utilizou a metodologia energética para analisar o balanço energético da lavoura cafeeira.

Também é possível afirmar que o sistema de produção agroquímico apresentou uma produtividade acumulada maior que o orgânico, mas o orgânico apresentou melhor eficiência. Como o manejo e a manutenção de cafezais orgânicos devem ser realizados de forma constante e intensiva, pode-se concluir que o sistema de produção orgânico é melhor alternativa de cultivo em relação ao sistema agroquímico.

Os fatores preponderantes na produção de café estão relacionados com a produtividade ao longo do tempo, portanto, conforme análises matemáticas e estatísticas o sistema orgânico, agrega maior valor, ao longo do tempo e ainda poderá ultrapassar a produtividade do sistema de produção agroquímico, proporcionando assim vantagens econômico-financeiras e ambientais ao produtor. Portanto, é de grande importância incentivar e apoiar agricultores a produzir café orgânico.

\section{REFERÊNCIAS}

ASSOCIAÇÃO DOS PRODUTORES ORGÂNICOS DE GARÇA E REGIÃO. Projeto Sebrae de produção de café orgânico. 2006. Disponível em: <http://www.garcafe.com.br/>. Acesso em 01/ago/2010.

BORGES FILHO, E. L. Da redução de insumos agrícolas à agroecologia: a trajetória das pesquisas com práticas agrícolas mais ecológicas na EMBRAPA. 2005. 279 f. Tese (Doutorado) - Instituto de Economia, Universidade Estadual de Campinas, Campinas, 2005.

CONAB - COMPANHIA NACIONAL DE ABASTECIMENTO. Planilhas de cálculo: custo de café. 2008. Disponível em: < http://www.conab.gov.br/>. Acesso em 01/ago/2010.

GUJARATI, D. N. Econometria básica. 3. ed. São Paulo: Pearson Education do Brasil, 2000. 548 p.

MACEDO, J. L. V.; PEREIRA, M. M. Análise financeira de sistemas agroflorestais em áreas abandonadas na Amazônia Ocidental. In; CONGRESSO BRASILEIRO DE SISTEMAS AGROFLORESTAIS, 3. Manaus. Resumos expandidos... Manaus: Embrapa Amazônia Oriental, 2000. p. 414-415.

MATOS, O. C. Econometria básica, 3. ed., São Paulo: Atlas. 2000. 438 p.

ORIGIN DATA ANALYSIS AND GRAPHING SOFTWARE. Disponível em: 
$<$ http://www.originlab.com/>. Acesso em 01/ago/2010.

ORTEGA, E. Introdução aos diagramas de fluxo de energia em ecossistemas, conceitos básicos de eficiência sistêmica e fórmulas de calculo energético que serão utilizadas no diagnóstico sócioambiental. Faculdade de Engenharia de Alimentos, Unicamp. Fevereiro de 2002. http://www.fea.unicamp.br/ortega/plan-disc/TA530-1a.htm

PENEIREIRO, F.M.; RODRIGUES, F.Q.; LUDEWIGS, T. 2000. Avaliação da sustentabilidade de sistemas agroflorestais no leste do Estado do Acre. In: CONGRESSO BRASILEIRO DE SISTEMAS AGROFLORESTAIS. 3, Manaus. Resumos expandidos... Manaus: Embrapa Amazônia Oriental, 2000. p.427-429.

PETERSEN.P; ALMEIDA. E. 2008. Revendo o conceito de fertilidade: conversão ecológica do sistema de manejo dos solos na região do Contestado, PB. Revista Agriculturas, v. 5, n.3. p.16-23.

REIS, C. S.; HILDEBRAND, M. Z. Avaliação da viabilidade de implantação de sistemas agroflorestais voltados para pequenas propriedades. In: CONGRESSO BRASILEIRO DE SISTEMA AGROFLORESTAIS, 3, Manaus. Resumos expandidos... Manaus: Embrapa Amazônia Oriental, 2000. p. 430-432.

ROMEIRO, A. R. Meio ambiente e dinâmica de inovações na agricultura. São Paulo: FAPESP. 1998. $277 \mathrm{p}$.

VEIGA, J. E. da. A agricultura no mundo moderno: diagnóstico e perspectivas. In: TRIGUEIRO, A (Org.). Meio ambiente no século 21: 21 especialistas falam da questão ambiental nas suas áreas de conhecimento. Rio de Janeiro: Sextante, 2003. p. 199-213. 